\title{
Energy substitutions, climate change and carbon sinks
}

\author{
Gilles Lafforgue*, Bertrand Magné ${ }^{\dagger}$ \\ and \\ Michel Moreaux
}

January 23, 2007

\begin{abstract}
We determine the optimal exploitation time-paths of two energy resources, one being depletable and polluting, namely a fossil fuel, the other being renewable and clean. These optimal paths are considered along with the two following features. First, the cumulative atmospheric pollution stock is set not to exceed some critical threshold and second, the polluting emissions produced by the use of fossil fuel can be reduced at the source and stockpiled in several carbon sinks of limited capacity. We show that, if the renewable resource flow is abundant, optimal sequestration only has to be implemented once the ceiling is reached. Moreover, the reservoirs should be completely filled by increasing order of their respective sequestration costs.
\end{abstract}

${ }^{*}$ University of Toulouse 1 (INRA and LERNA), 21 Allée de Brienne, 31000 Toulouse, France. E-mail: glafforg@toulouse.inra.fr.

${ }^{\dagger}$ Paul Scherrer Institute (LEA), CH-5232, Villigen, PSI, Switzerland. E-mail: bertrand.magne@psi.ch.

${ }^{\ddagger}$ University of Toulouse I (IUF, IDEI and LERNA), 21 Allée de Brienne, 31000 Toulouse, France. 


\section{Introduction}

Greenhouse gases emissions essentially result from the use of fossil, carbon-based, energy resources ${ }^{1}$. Numerous technical and regulatory devices allowing for emission abatement are readily available. Their implementation may arise sooner or later depending on their respective costs. One of the now commonly advocated mean to mitigate the atmospheric pollution consists in capturing the dirty particles at the source of emission and storing it underground, either in natural reservoirs or in depleted mine sites that will be called carbon sinks in the remainder of the text ${ }^{2,3}$.

In the present study, we first intend to determine the optimal starting date and pace of this carbon sequestration mode that has been the focus of a recent IPCC $^{4}$ special report (IPCC, 2005). Second, we want to characterize how the recourse to such an abatement option on the flow of pollution emissions alters the optimal time path of fossil depletable resources, when the cumulative atmospheric concentration of carbon has to be maintained below some given critical threshold, in accordance to the Kyoto Protocol ${ }^{5}$.

The possibility of sequestering some fraction of the carbon dioxide emitted by the combustion of fossil resources has motivated numerous empirical studies (see for example the results of integrated assessment models from Mc Farland et al., 2003, Edmonds et al., 2004, Kurosawa, 2004, Gerlagh 2006, Gerlagh R. and van der Zwaan, 2006, Gitz et al., 2005, Riahi et al., 2004). The level of complexity of such operational models, aimed at defining some climate policy, may be required so as to take into account the numerous

\footnotetext{
${ }^{1}$ Among others, animal and human wastes, the reduction of forest cover and the extensive agricultural practices also constitute non negligible emissions sources and favor carbon release from shallow soils.

${ }^{2}$ The carbon sinks also refer to biomass carbon storage, e.g. in soils, plants and especially trees (See for example IPCC, 2001). Biomass carbon storage is not the focus of the present analysis.

${ }^{3}$ Captured gases may also be injected again in oil deposits so as to enhance the oil recovery, and thus increasing the oil resource base. This process is already being operated in the North Sea by the Norwegian oil company Statoil. The economic analysis of such a storage process poses some specific problems that are here eluded.

${ }^{4}$ Intergovernmental Panel on Climate Change.

${ }^{5}$ Even though the ultimate goal of the IPCC consists in stabilizing the atmospheric concentration on greenhouse gases, the Kyoto Protocol does not prescribe the level of such a target. It rather prescribes some constraint on the maximum flow of carbon emissions for the industrialized countries. More precisely, their emissions shall be reduced down to their levels of the reference year 1990, at the end of the commitment period i.e. year 2012. Would an atmospheric stabilization target be set, this kind of agreement would remain ineffective until each participating countries' effort has not been set. This classical question of the cost of a public good and its breakdown among agents is made more complex in the present setting because of the dynamic nature of the problem. Since the key variable is the accumulation of greenhouse gases (we will restrain our analysis to the case of carbon dioxide), the most straightforward way of tackling the problem is to set an upper bound on the state variable.
} 
interactions at hand. We here take a radically different approach and use a highly stylized model so as to exhibit the main driving forces that usually tend to be blurred by the multiple retroactions of more complex settings.

To go straight to the point, we assume that there are final users that consume two primary energies: a polluting and scarce fossil resource, and a clean and renewable backstop resource. The users derive their utility from the use of energy in its final form, and thus face an energy cost that comprises all the costs incurred through the transformation process of the primary energy source. With this respect, the two types of energies are perfect substitutes for the final users. Moreover, in accordance with current technologies, we assume that the useful energy obtained from the non renewable resource is cheaper than the energy obtained from the renewable one.

To assume the existence of an atmospheric concentration ceiling, this pollution stock being in addition partially eliminated owing to natural decay or absorption ${ }^{6}$, implicitly constrains the instantaneous rate of consumption of the polluting resource once the threshold is reached, absent any pollution capture and storage option. Two observations then deserve to be mentioned.

First, along the optimal path, the time interval during which the flow of fossil consumption is constrained is endogenous. The carbon ceiling is reached at a date that is a function of the fossil consumption path from the initial time period on. As a consequence, the cap imposed on the carbon accumulation affects the entire time path of the fossil resource exploitation, as well as the one of the backstop because they are perfect substitutes.

Second, two options are available to the society if she wants to relax the constraint on fossil fuel use once at the ceiling. She may either substitute the dirty resource for the clean one, or sequester some fraction of the polluting flow generated by the fossil resource use. Each of those options entails some monetary cost. The renewable resource is more expensive than the non-renewable one. To capture the carbon dioxide is also costly. But each of these options also exhibits some specific opportunity cost.

\footnotetext{
${ }^{6}$ This process can be interpreted as some natural carbon sequestration by a sink of very large size, e.g. typically the oceans (For more details, see IPPC, 2001).
} 
A scarcity rent is to be associated to the consumption of the fossil resource, as is the case for every non-renewable resource. To this rent augmented by the extraction and processing cost of the fossil, we must add now the shadow cost of the atmospheric carbon stock and, when pollution is abated at the source of emissions, the cost of capturing the carbon augmented by the shadow cost of the $\operatorname{sink}^{7}$. To set down a cap on the accumulation of pollution, possibly removable by sequestration, results in having de facto two carbon storage deposits at disposal. The first one is the atmospheric reservoir of temporary bounded capacity, but of infinite long run capacity thanks to the natural progressive regeneration. But to take advantage of this infinite long run capacity, the carbon emission flow has to be restrained when the temporary capacity is saturated. Hence some rent has to be charged for the use of this capacity, even before it is reached as shall show. The second reservoir is the sink, which itself can be of limited capacity so that another rent must be charged for its use. Clearly at each point of time, some part of the emission flow can be sent in some reservoir, thus having to bear the corresponding rent, while the other part is sent into the other reservoir, having to bear the other rent. The dynamics of these two rents obey two different rules and their trajectories diverge over time. Note that the renewable resource would also entail a scarcicity rent if the exploitable carbon-free flow is not sufficiently abundant. For the sake of simplicity, we assume here that the renewable substitute is abundant.

In order to characterize the dynamics of those various rents, we assume that all the marginal monetary costs are constant over time, as well as the natural regeneration rate, the utility of energy consumption and the generated surplus functions.

The article is organized as follows. Section 2 presents the model. In section 3, we determine the optimal exploitation time-paths of the two resources if sequestration is not allowed. In section 4, we examine the optimal paths when the stockpiling capacity of a single sink is large enough so as to store as much carbon as needed. Such carbon sinks are said to be "large". We show in section 5 how the optimal trajectories are altered when the carbon deposit is "small". The size of the deposit, either small or large, is thus an endogenous characteristic. The way the results are ordered can be seen as an algorithm

\footnotetext{
${ }^{7}$ With this respect, the problem is similar to the case of industrial and residential wastes management treated in Gaudet et al. (2001), but here the cost of waste before any dispensing into some discharge must include the Hotelling rent mine (Hotelling, 1931). Note also that, contrary to Gaudet et al. (2001), we do not assume any sunk fixed cost for activating the discharge.
} 
aimed at determining whether a deposit is small or large. We generalize our analysis to the case of multiple storage deposits that are differentiated by their access costs and their storage capacities. We show that those deposits have to be exploited by increasing order of access cost, no matter their capacity, and we identify the most costly deposit having to be used. We also show how this multiple deposits model can be interpreted as the approximation of a more realistic model where the cost of sequestration in each deposit is an increasing function of the cumulative stored carbon. We briefly conclude in section 6 .

\section{The model}

\subsection{Assumptions and notations}

We consider an economy in which the instantaneous gross surplus, or utility, generated by an instantaneous energy consumption ${ }^{8} q_{t}$ is given by $u\left(q_{t}\right)$, where function $u($.$) has the$ following standard properties:

Assumption (A.1): $u: \mathbb{R}_{++} \rightarrow \mathbb{R}_{+}$is a function of class $\mathcal{C}^{2}$ strictly increasing and strictly concave, satisfying the Inada conditions: $\lim _{q \downarrow 0} u^{\prime}(q)=+\infty$, where $u^{\prime}(q) \equiv$ $d u / d q$.

We also use $p$ to denote the marginal surplus $u^{\prime}$ as well as, by a slight abuse of notation, the marginal surplus function: $p(q)=u^{\prime}(q)$. The direct demand function $d(p)$ is the inverse of $p(q)$, as usually defined. We denote by $u^{\prime \prime}$ the second derivative of $u$. Under (A.1), $u^{\prime}(q)>0$ and $u^{\prime \prime}(q)<0, \forall q>0$.

Energy needs may be supplied by two resources, either a dirty non-renewable resource, a fossil resource such as coal, or a clean renewable resource, such as solar energy. We assume that these two energy sources are perfect substitutes, so that if $x_{t}$ denotes the instantaneous consumption of the fossil resource and $y_{t}$, the instantaneous consumption of the renewable resource, then the surplus generated by the total consumption $\left(x_{t}, y_{t}\right)$ is $u\left(x_{t}+y_{t}\right)$, i.e. $q_{t}=x_{t}+y_{t}$.

The average cost of transforming coal into directly usable energy is constant and equal to $c_{x}$, hence $c_{x}$ is also the constant marginal $\operatorname{cost}^{9}$. Let $\tilde{x}$ denote the flow of fossil resource

\footnotetext{
${ }^{8}$ Strictly speaking, $q_{t}$ is a power, so assuming that $q_{t}$ is differentiable, the energy consumed over a time interval $[t, t+d t]$ is equal to $\left(q_{t}+\dot{q}_{t}\right) d t$, where $\dot{q}_{t}=d q_{t} / d t$.

${ }^{9}$ This cost include all the processing costs for delivery to the final users.
} 
to be consumed in order to equalize the marginal surplus to the marginal monetary cost of the resource. Thus, $\tilde{x}$ is the solution of $u^{\prime}(x)=c_{x}$, that is, $\tilde{x}=d\left(c_{x}\right)$.

Let $X^{0}$ be the initial fossil resource stock and $X_{t}$, the stock available at time $t$, so that:

$$
\dot{X}_{t}=-x_{t}, X_{0}=X^{0}, X_{t} \geq 0 \text { and } x_{t} \geq 0, t>0 .
$$

Using coal potentially generates a pollutant flow. Let $\zeta$ be the unitary carbon content of the fossil resource so that, without any abatement policy, the instantaneous carbon flow released into the atmosphere would be equal to $\zeta x_{t}$.

Let $Z_{t}$ be the stock of pollutant in the atmosphere at time $t, z_{t}$ the flow of emissions and $\alpha, \alpha>0$, the instantaneous proportional rate of natural regeneration, assumed to be constant for the sake of simplicity (see for instance Kolstad and Krautkraemer, 1993) so that:

$$
\dot{Z}_{t}=z_{t}-\alpha Z_{t}
$$

We assume that this stock of carbon cannot be larger than some threshold $\bar{Z}$. Let $Z^{0}$ the stock of carbon in the atmosphere at the beginning of the planning period, assumed to be smaller than $\bar{Z}$. We thus have:

$$
\bar{Z}-Z_{t} \geq 0, t \geq 0 \text { and } Z_{0}=Z^{0}
$$

However, let us assume that some carbon sequestration device is available. The potential pollution flow can be reduced at the source of emission and stockpiled in $n$ carbon sinks, or reservoirs, indexed by $i, i=1, \ldots, n$. Each $\operatorname{sink} i$ is characterized by its unitary sequestration cost $c_{s i}$ and its capacity $\bar{S}_{i}$. By convention, the $n$ reservoirs are ranked by strictly increasing order of costs: $c_{s 1}<\ldots<c_{s i}<\ldots<c_{s n}$. Capacities and sequestration costs are independent a priori and the theory developed here remains compatible with any form of relationship. Let $S_{i}^{0}$ be the initial stock of pollutant in reservoir $i$. Without any loss of generality, we postulate that $S_{i}^{0}=0, i=1, \ldots, n$. We thus have:

$$
z_{t}=\zeta x_{t}-\sum_{i=1}^{n} s_{i t}, t \geq 0
$$

where $s_{i t}$ is the part of the potential carbon emission flow that is sequestered into reservoir $i$ at time $t$, so that:

$$
\begin{array}{ll}
\dot{S}_{i t}=s_{i t}, S_{i 0}=0 \text { and } s_{i t} \geq 0 & , i=1, \ldots, n \text { and } t \geq 0 \\
\bar{S}_{i}-S_{i t} \geq 0 & , i=1, \ldots, n \text { and } t \geq 0 .
\end{array}
$$


Once the ceiling $\bar{Z}$ is reached, we must have $\dot{Z}_{t}=0$. Then, the flow of fossil resource that could be consumed at the ceiling without any sequestration scheme is $\bar{x}=\alpha \bar{Z} / \zeta$. We use $\bar{p}$ to denote the marginal utility of $\bar{x}: \bar{p}=u^{\prime}(\bar{x})$. If sequestration in reservoir $i$ needs to be implemented, we postulate that the total marginal cost of a "clean" consumption of the fossil resource, $c_{x}+c_{s i} \zeta$, is lower than $\bar{p}$. If not, we would always be better off remaining at $\bar{x}$ rather than relaxing the constraint by sequestering any part of the emission flow in reservoir $i$.

Assumption (A.2): $\forall i=1, \ldots, n: c_{x}+c_{s i} \zeta<\bar{p}$.

The other resource is a non-polluting renewable resource that can be made available to the end users at a constant average cost $c_{y}$. The cost of the renewable resource is the total cost of supplying the good to the final users, so that the non-renewable and the renewable resources are perfect substitutes for the users. We assume that $c_{y}>\bar{p}$ since, if $c_{y}$ was lower than $\bar{p}$, condition $\bar{Z}-Z_{t}$ would never constrain the energy consumption.

Let $\bar{y}$ be the constant instantaneous flow of this renewable energy available at each point of time. We denote by $\tilde{y}$ the flow of renewable resource that the society would have to consume once the fossil resource is exhausted, provided that $\bar{y}$ is sufficiently large. Then, $\tilde{y}$ is the solution of $u^{\prime}(y)=c_{y}: \tilde{y}=d\left(c_{y}\right)$. We assume that the available flow of the renewable resource is abundant, i.e. at least equal to $\tilde{y}$, so that no rent has to be charged for the use of this resource ${ }^{10}$.

Assumption (A.3): $\bar{p}<c_{y}$ and $\tilde{y}<\bar{y}$.

Let us assume that the instantaneous social rate of discount, $\rho>0$, is constant. The objective of the social planner is to choose the resource and sequestration trajectories that maximize the sum of the discounted instantaneous net surplus.

\subsection{Problem formulation and optimality conditions}

The social planner problem $(P)$ can be expressed as follows:

$$
\max _{\left\{\left(s_{i t}, i=1, \ldots, n, x_{t}, y_{t}\right), t \geq 0\right\}} \int_{0}^{\infty}\left[u\left(x_{t}+y_{t}\right)-\sum_{i=1}^{n} c_{s i} s_{i t}-c_{x} x_{t}-c_{y} y_{t}\right] e^{-\rho t} d t
$$

\footnotetext{
${ }^{10}$ The case of a constrained flow of renewable resource is analyzed in Lafforgue et al. (2005).
} 
subject to constraints:

$$
\begin{array}{lr}
\dot{X}_{t}=-x_{t}, X_{0}=X^{0}>0 \text { given } & \\
X_{t} \geq 0 & \\
\dot{S}_{i t}=s_{i t}, S_{i 0}=0, & i=1, \ldots, n \\
\bar{S}_{i}-S_{i t} \geq 0, & i=1, \ldots, n \\
\dot{Z}_{t}=\zeta x_{t}-\sum_{i=1}^{n} s_{i t}-\alpha Z_{t}, Z_{0}=Z^{0}<\bar{Z} \text { given } & \\
\bar{Z}-Z_{t} \geq 0 & \\
\zeta x_{t}-\sum_{i=1}^{n} s_{i t} \geq 0 & \\
s_{i t} \geq 0, & \\
x_{t} \geq 0 \text { and } y_{t} \geq 0 . &
\end{array}
$$

Let $\mathcal{L}_{t}$ be the current valued Lagrangian for the problem $(P)^{11}$ :

$$
\begin{aligned}
\mathcal{L}_{t}= & u\left(x_{t}+y_{t}\right)-\sum_{i=1}^{n} c_{s i} s_{i t}-c_{x} x_{t}-c_{y} y_{t}-\lambda_{t} x_{t}+\sum_{i=1}^{n} \eta_{i t} s_{i t} \\
& +\mu_{t}\left[\zeta x_{t}-\sum_{i=1}^{n} s_{i t}-\alpha Z_{t}\right]+\sum_{i=1}^{n} \nu_{i t}\left[\bar{S}_{i}-S_{i t}\right]+\nu_{Z t}\left[\bar{Z}-Z_{t}\right] \\
+ & \bar{\gamma}_{s t}\left[\zeta x_{t}-\sum_{i=1}^{n} s_{i t}\right]+\sum_{i=1}^{n} \gamma_{i t} s_{i t}+\gamma_{x t} x_{t}+\gamma_{y t} y_{t} .
\end{aligned}
$$

The first-order conditions of $(P)$ are:

$$
\begin{aligned}
\partial \mathcal{L}_{t} / \partial s_{i t}=0 & \Leftrightarrow \quad c_{s i}=\eta_{i t}-\mu_{t}-\bar{\gamma}_{s t}+\gamma_{i t}, \quad i=1, \ldots, n \\
\partial \mathcal{L}_{t} / \partial x_{t}=0 & \Leftrightarrow \quad u^{\prime}\left(x_{t}+y_{t}\right)=c_{x}+\lambda_{t}-\mu_{t} \zeta-\bar{\gamma}_{s t} \zeta-\gamma_{x t} \\
\partial \mathcal{L}_{t} / \partial y_{t}=0 & \Leftrightarrow \quad u^{\prime}\left(x_{t}+y_{t}\right)=c_{y}-\gamma_{y t},
\end{aligned}
$$

with the following associated complementary slackness conditions:

$$
\begin{array}{rlll}
\bar{\gamma}_{s t} \geq 0 \quad \text { and } & \bar{\gamma}_{s t}\left[\zeta x_{t}-\sum_{i=1}^{n} s_{i t}\right]=0 & \\
\gamma_{i t} \geq 0 & \text { and } & \gamma_{i t} s_{i t}=0, & \\
\gamma_{x t} \geq 0 & \text { and } & \gamma_{x t} x_{t}=0 \\
\gamma_{y t} \geq 0 & \text { and } & \gamma_{y t} y_{t}=0 .
\end{array}
$$

\footnotetext{
${ }^{11}$ As is customary in such problems, we neglect the constraint $X_{t} \geq 0$ in the Lagrangian expression.
} 
The dynamics of the co-state variables must verify:

$$
\begin{aligned}
\dot{\lambda}_{t}=\rho \lambda_{t}-\partial \mathcal{L}_{t} / \partial X_{t} & \Leftrightarrow \quad \dot{\lambda}_{t}=\rho \lambda_{t} \Leftrightarrow \lambda_{t}=\lambda_{0} e^{\rho t} \\
\dot{\eta}_{i t}=\rho \eta_{i t}-\partial \mathcal{L}_{t} / \partial S_{i t} & \Leftrightarrow \quad \dot{\eta}_{i t}=\rho \eta_{i t}+\nu_{i t}, \quad i=1, \ldots, n \\
\dot{\mu}_{t}=\rho \mu_{t}-\partial \mathcal{L}_{t} / \partial Z_{t} & \Leftrightarrow \quad \dot{\mu}_{t}=(\alpha+\rho) \mu_{t}+\nu_{Z t},
\end{aligned}
$$

with the following complementary slackness conditions:

$$
\begin{aligned}
& \nu_{i t} \geq 0 \quad \text { and } \quad \nu_{i t}\left[\bar{S}_{i}-S_{i t}\right]=0, \quad i=1, \ldots, n \\
& \nu_{Z t} \geq 0 \quad \text { and } \quad \nu_{Z t}\left[\bar{Z}-Z_{t}\right]=0 \text {. }
\end{aligned}
$$

The other transversality conditions write:

$$
\begin{array}{ll}
\lim _{t \uparrow \infty} e^{-\rho t} \lambda_{t} X_{t}=\lambda_{0} \lim _{t \uparrow \infty} X_{t}=0 & \\
\lim _{t \uparrow \infty} e^{-\rho t} \eta_{i t} S_{i t}=0, & i=1, \ldots, n \\
\lim _{t \uparrow \infty} e^{-\rho t} \mu_{t} Z_{t}=0 . &
\end{array}
$$

First, we note that $\eta_{i t} \leq 0, \eta_{i t}$ being the instantaneous marginal value of the carbon stock which is already sequestered into $\operatorname{sink} i$ at time $t$. If the storage capacity of this sink was limited and if at date $t, S_{i t}$ was increased by an exogenous amount $d S_{i t}>0$, then the optimal value of the objective function of $(P)$ would diminish ${ }^{12}$. Second, as long as reservoir $i$ is not completely filled, i.e. for any $t$ such that $\bar{S}_{i}-S_{i t}>0, \nu_{i t}=0$, so that from (19) it comes:

$$
\bar{S}_{i}-S_{i t}>0 \Rightarrow \eta_{i t}=\eta_{i 0} e^{\rho t}
$$

By the same type of argument, $\mu_{t} \leq 0, \mu_{t}$ being the instantaneous marginal value of the carbon stock in the atmosphere at date $t$. Since the stock of carbon in the atmosphere is also limited, if at date $t, Z_{t}$ was increased by an exogenous amount $d Z_{t}>0$, the optimal value of the objective function of $(P)$ would, at best, remain constant, and would decrease in the worst case. Furthermore, since $Z^{0}<\bar{Z}$, there must exist an initial time interval during which the stock of carbon in the atmosphere is below the ceiling, hence $\nu_{Z t}=0$ so that integrating (20), we get:

$$
\mu_{t}=\mu_{0} e^{(\alpha+\rho) t} .
$$

The difference in the dynamics of $\eta_{i t}, i=1, \ldots, n$ and of $\mu_{t}$ can be explained as follows. On the one hand, the decision to resort to the storage capacity of reservoir $i$ is irreversible

\footnotetext{
${ }^{12}$ If the capacity of the sink is sufficiently large, then $\eta_{i t}=0, t \geq 0, c f$. section 4 .
} 
since $S_{i t}$ is implicitly a monotonous and non-decreasing function. On the other hand, owing to the natural regeneration, the stock of pollutant in the atmosphere $Z_{t}$ can either increase or decrease. On that account, whatever the sequestration policy may be, $Z_{t}$ will be induced to decrease since the fossil resource stock is finite. As it will be seen in the next sections, such an asymmetry strongly governs the optimal solution.

Finally, if there exists $\bar{t}$ such that for any $t \geq \bar{t}$ the ceiling constraint is no more binding forever, then $\mu_{t}=0, t \geq \bar{t}$.

\section{$3 \quad$ Hotelling and optimal paths without any carbon sink}

\subsection{Herfindahl-Hotelling paths}

Absent the ceiling constraint, the optimal solution would be the well-known HerfindahlHotelling path ${ }^{13,14}$.

For any $\lambda_{0} \in\left(0, c_{y}-c_{x}\right)$, define $p_{t}^{H}\left(\lambda_{0}\right)$ as the price path along which the coal rent is increasing at the discount rate $\rho, p_{t}^{H}\left(\lambda_{0}\right)=c_{x}+\lambda_{0} e^{\rho t} ; T^{H}\left(\lambda_{0}\right)$ as the time at which $p_{t}^{H}\left(\lambda_{0}\right)$ is choking the average cost of the abundant renewable substitute, $T^{H}\left(\lambda_{0}\right)=$ $\frac{1}{\rho}\left[\ln \left(c_{y}-c_{x}\right)-\ln \lambda_{0}\right] ; d_{t}^{H}\left(\lambda_{0}\right)$ as the corresponding instantaneous energy demand, $d_{t}^{H}\left(\lambda_{0}\right) \equiv$ $d\left(p_{t}^{H}\left(\lambda_{0}\right)\right)$ and $z_{t}^{H}\left(\lambda_{0}\right)$ as the generated polluting flow, $z_{t}^{H}\left(\lambda_{0}\right)=\zeta d_{t}^{H}\left(\lambda_{0}\right)$; last $D_{t}^{H}\left(\lambda_{0}\right)$ as the cumulative demand, $D_{t}^{H}\left(\lambda_{0}\right)=\int_{0}^{t} d_{\tau}^{H}\left(\lambda_{0}\right) d \tau$.

Trivial calculations would show that $\bar{D}^{H}\left(\lambda_{0}\right) \equiv D_{T^{H}\left(\lambda_{0}\right)}^{H}\left(\lambda_{0}\right)$ is a strictly decreasing function with $\lim _{\lambda_{0} \downarrow 0} D^{H}\left(\lambda_{0}\right)=+\infty$ and $\lim _{\lambda_{0} \uparrow\left(c_{y}-c_{x}\right)} D^{H}\left(\lambda_{0}\right)=0$, so that the equilibrium equation:

$$
\bar{D}^{H}\left(\lambda_{0}\right)=X^{0}
$$

has a unique solution $\lambda_{0}^{H}$ which is the optimal value of $\lambda_{0}$.

Thus the optimal path is a two phases path. During the first phase $\left[0, T^{H}\left(\lambda_{0}^{H}\right)\right]$, the energy demand is supplied only by the fossil fuel, and the energy consumption is decreasing

\footnotetext{
${ }^{13}$ Herfindahl (1967), Hotelling (1931).

${ }^{14}$ Without such a constraint the objective function of program $(P)$ writes:
}

$$
\int_{0}^{\infty}\left[u\left(x_{t}+y_{t}\right)-c_{x} x_{t}-c_{y} y_{t}\right] e^{-\rho t} d t
$$

and the constraints (4)-(9) vanish. The first order condition (11), the conditions (14), (15), (19)-(22) and (24)-(25) also vanish and, from (18), the condition (12) becomes:

$$
u^{\prime}\left(x_{t}+y_{t}\right)=c_{x}+\lambda_{0} e^{\rho t}-\gamma_{x t} .
$$

The conditions (13), (16), (17) and (23) remain the same. 
from $d_{0}^{H}\left(\lambda_{0}\right)=d\left(c_{x}+\lambda_{0}\right)$ down to $d_{T^{H}\left(\lambda_{0}^{H}\right)}^{H}=d\left(c_{y}\right)$. From $T^{H}\left(\lambda_{0}^{H}\right)$ onwards, the energy consumption is stationary and equal to $d\left(c_{y}\right)$, supplied by the renewable source.

The pollution emission flow is commanding the pollution stock trajectory. Let $Z_{t}^{H}\left(\lambda_{0}\right)$ be the pollution stock induced by the emission flow:

$$
z_{t}^{H}\left(\lambda_{0}\right)= \begin{cases}\zeta d_{t}^{H}\left(\lambda_{0}\right) & , \quad t \in\left[0, T^{H}\left(\lambda_{0}\right)\right) \\ 0 & , \quad t \in\left[T^{H}\left(\lambda_{0}\right),+\infty\right)\end{cases}
$$

that is ${ }^{15}$ :

$$
Z_{t}^{H}\left(\lambda_{0}\right)= \begin{cases}Z^{0} e^{-\alpha t}+\zeta \int_{0}^{t} d_{\tau}^{H}\left(\lambda_{0}\right) e^{-\alpha(t-\tau)} d \tau & , t \in\left[0, T^{H}\left(\lambda_{0}\right)\right) \\ Z^{0} e^{-\alpha t}+e^{-\alpha\left(t-T^{H}\left(\lambda_{0}\right)\right)} \zeta \int_{0}^{T^{H}\left(\lambda_{0}\right)} d_{\tau}^{H}\left(\lambda_{0}\right) e^{-\alpha(t-\tau)} d \tau & , t \in\left[T^{H}\left(\lambda_{0}\right),+\infty\right) .\end{cases}
$$

Let $\bar{Z}^{H}\left(\lambda_{0}\right) \equiv \max \left\{Z_{t}^{H}\left(\lambda_{0}\right), t \geq 0\right\}$. Clearly:

$$
\frac{d \bar{Z}^{H}\left(\lambda_{0}\right)}{d \lambda_{0}}<0
$$

and there exists some critical value $\bar{X}^{0}$ of $X^{0}$ such that:

$$
\bar{Z}^{H}\left(\bar{X}^{0}\right)=\bar{Z} \quad \text { and } \quad \bar{D}^{H}\left(\lambda_{0}\right)=\bar{X}^{0} .
$$

For $X^{0}<\bar{X}^{0}$, the ceiling constraint is never active, $Z_{t}^{H}\left(\lambda_{0}^{H}\right)<\bar{Z}$, and for $X^{0}>\bar{X}^{0}$ there must exist some time interval within which $Z_{t}^{H}\left(\lambda_{0}^{H}\right)>\bar{Z}$ thus violating the constraint. We assume from now that the initial amount of fossil fuel is sufficiently large in such a way that, without any ceiling constraint, the critical level of the pollution stock would be overshot over some time interval.

Assumption (A.4): $X^{0}>\bar{X}^{0}$.

\subsection{Optimal paths when $X^{0}>\bar{X}^{0}$, without any abatement opportunity}

Under (A.4) and with no active abatement opportunity, it is well known that the optimal consumption path is the four-phase path ${ }^{16}$ illustrated in Figure 1.

\footnotetext{
${ }^{15}$ At time $t, Z_{t}^{H}\left(\lambda_{0}\right)$ is the sum of $Z^{0} e^{-\alpha t}$, this part of the initial pollution stock not yet naturally regenerated at this time, and the different $\zeta d_{\tau}^{H}\left(\lambda_{0}\right) e^{-\alpha(t-\tau)}, \tau \in[0, t]$, this part of the emission flow of time $\tau$ not yet regenerated. After $T^{H}\left(\lambda_{0}\right)$, there is no new emission and the stock smoothly decline according to $e^{-\alpha\left(t-T^{H}\left(\lambda_{0}\right)\right)} Z_{T^{H}\left(\lambda_{0}\right)}^{H}\left(\lambda_{0}\right)$, done to 0 .

${ }^{16}$ See Chakravorty et al. $(2006-\mathrm{a}, 2006-\mathrm{b})$ for detailed studies of these paths.
} 
During a first phase $\left[0, t_{1}\right)$, the constraint is slack and only the fossil resource has to be used: $q_{t}=x_{t}=d\left(c_{x}+\lambda_{0} e^{\rho t}-\mu_{0} e^{(\alpha+\rho) t} \zeta\right)$, with $\lambda_{0}$ and $\left|\mu_{0}\right|$ are sufficiently low so that $x_{t}>\bar{x}$. Since $x_{t}>\bar{x}$ and $Z_{t}<\bar{Z}$, the flow of pollutant emissions $\zeta x_{t}$ is higher than the natural regeneration flow $\alpha Z_{t}$ and $Z_{t}$ increases. At the end of the phase, at $t_{1}$, the carbon ceiling is reached and the full marginal cost of the fossil resource, $c_{x}+\lambda_{0} e^{\rho t}-\mu_{0} e^{(\alpha+\rho) t} \zeta$, is equal to $\bar{p}$.

The second phase $\left[t_{1}, t_{2}\right)$ is a constrained phase at the ceiling. The fossil consumption is bounded by $\bar{x}, q_{t}=x_{t}=\bar{x}$ and the energy price is constant and equal to $\bar{p}$. Since $\bar{p}=c_{x}+\lambda_{0} e^{\rho t}-\mu_{t} \zeta$, then $\left|\mu_{t}\right|$ must be decreasing during this phase. At $t_{2}, \mu_{t}=0$ and the ceiling constraint will no longer be active so that $\mu_{t}$ is nil from $t_{2}$ onwards.

The third phase $\left[t_{2}, t_{3}\right)$ is a pure Hotelling phase during which only the fossil resource is used: $p_{t}=p_{t}^{H}\left(\lambda_{0}\right)=c_{x}+\lambda_{0} e^{\rho t}$ and $q_{t}=d_{t}^{H}\left(\lambda_{0}\right)=x_{t}=d\left(c_{x}+\lambda_{0} e^{\rho t}\right)$. The fossil resource consumption thus decreases and the stock is exhausted at the end of the phase. At $t_{3}$, the price $p_{t}^{H}\left(\lambda_{0}\right)$ is equal to the marginal cost of the renewable resource $c_{y}$.

During the last phase $\left[t_{3}, \infty\right)$, only the renewable resource is consumed, $q_{t}=y_{t}=\tilde{y}$ and $p_{t}=c_{y}$.

Figure 1 here

The optimal values of the five fundamental variables $\lambda_{0}, \mu_{0}, t_{1}, t_{2}$ and $t_{3}$ are determined as the solution of a five-equation system detailed in Appendix A.1.

\section{The case of a single large reservoir}

In this section, we assume that the sequestration device consists in a single carbon $\operatorname{sink}^{17}$ and that the capacity of the sink is "large". Large capacity is an endogenous characteristic

\footnotetext{
program writes now:

$$
\int_{0}^{\infty}\left[u\left(x_{t}+y_{t}\right)-c_{s} s_{t}-c_{x} x_{t}-c_{y} y_{t}\right] e^{-\rho t} d t
$$

Constraints (4)-(9) become:

$$
\begin{aligned}
& \dot{S}_{t}=s_{t}, S_{0}=S^{0}, \bar{S}-S_{t} \geq 0, \dot{Z}_{t}=\zeta x_{t}-s_{t}-\alpha Z_{t} \\
& \zeta x_{t}-s_{t} \geq 0 \text { and } s_{t} \geq 0
\end{aligned}
$$
}

${ }^{17}$ If there is only one reservoir, we can neglect the index on $s_{t}$ and $S_{t}$. The objective function of the 
of the sink that depends upon all the other fundamentals of the model, as it will be seen at the end of the section. For the moment, the reservoir capacity is said to be sufficiently large so that no rent has to be charged, meaning that its capacity will never an active constraint. Hence, the sequestration cost is $c_{s} \zeta$ for each unit of consumed fossil resource. Discounting implies that this cost must be borne as late as possible. Hence, it is optimal to sequester only once the stock of pollutant in the atmosphere is reaching the ceiling $\bar{Z}$.

We first note that for $\eta_{t}=0$ since the reservoir capacity constraint is never active. If furthermore some part of the carbon emissions is sequestered, i.e. $s_{t}>0$ implying that $\gamma_{s t}=0$, then the optimality condition (11) becomes:

$$
c_{s}=-\mu_{t}-\bar{\gamma}_{s t} \Rightarrow-\mu_{t}=c_{s}+\bar{\gamma}_{s t}
$$

Next, substituting the above value of $\mu_{t}$ into the optimality condition (12), bearing in mind $s_{t}>0$ implies that $x_{t}>0$, hence $\gamma_{x t}=0$, we obtain:

$$
u^{\prime}\left(x_{t}\right)=c_{x}+c_{s} \zeta+\lambda_{0} e^{\rho t}
$$

which, in turn, implies that:

$$
x_{t}=d\left(c_{x}+c_{s} \zeta+\lambda_{0} e^{\rho t}\right)
$$

If for any phase during which only the fossil resource is used, the ceiling constraint $\bar{Z}-Z_{t} \geq 0$ is active and a part of the carbon emissions is sequestered, then the resource consumption must be equal to $d\left(c_{x}+c_{s} \zeta+\lambda_{0} e^{\rho t}\right)$ so as to satisfy the first order conditions. However, it does not mean that the totality of the emission flow is necessarily captured. Minimization of costs implies that only part of emissions that exceeds the ceiling has to be stockpiled, so that:

$$
s_{t}=\zeta\left[d\left(c_{x}+c_{s} \zeta+\lambda_{0} e^{\rho t}\right)-\bar{x}\right] .
$$

Hence, that affects some first order conditions in the following way:

$\bar{\gamma}_{s t} \geq 0$ and $\bar{\gamma}_{s t}\left[\zeta x_{t}-s_{t}\right]=0$

(15) $\gamma_{s t} \geq 0$ and $\gamma_{s t} s_{t}=0$

(19) $\quad \dot{\eta}_{t}=\rho \eta_{t}+\nu_{S t}$

(21) $\quad \nu_{S t} \geq 0$ and $\nu_{S t}\left[\bar{S}-S_{t}\right]=0$

(24) $\quad \lim _{t \uparrow \infty} e^{-\rho t} \eta_{t} S_{t}=0$

and $\eta_{t}=\eta_{0} e^{\rho t}$ as long as $\bar{S}-S_{t}>0$. 
This equation implies firstly that $\gamma_{s t}=0$ since $s_{t}>0$, and $\gamma_{x t}=0$ since $x_{t}>0$, secondly that $\bar{\gamma}_{s t}=0$ because $\zeta x_{t}-s_{t}>0$, the emissions being not entirely sequestered. Thus we get from (11): $c_{s}=-\mu_{t}$, hence the expression (28) of condition (12) for those particular values of $-\mu_{t}, \bar{\gamma}_{s t}, \gamma_{s t}$ and $\gamma_{x t}$, and for $y_{t}=0$.

When $Z_{t}=\bar{Z}$, the full marginal cost of the energy satisfying the ceiling constraint is a two-step function, equal to the monetary extraction cost augmented by the rent if $x_{t} \leq \bar{x}$, at which the abatement $\operatorname{cost} c_{s} \zeta$ must be added if $x_{t}>\bar{x}$ :

$$
c_{m}= \begin{cases}c_{x}+\lambda_{0} e^{\rho t} & , \text { if } x_{t} \leq \bar{x} \\ c_{x}+\lambda_{0} e^{\rho t}+c_{s} \zeta & , \text { if } x_{t}>\bar{x}\end{cases}
$$

Figure 2 here

Figure 2 illustrates why, at the ceiling, it is optimal first to abate and to sequester and next not to abate. In Figure 3 the marginal cost curves are drawn for three dates $t, t^{\prime}$, $t^{\prime \prime}, t<t^{\prime}<t^{\prime \prime}$, sufficiently spaced but not too much, when the economy is at the ceiling. At date $t$, the inverse demand function is crossing the upper branch of the marginal cost curve at $x_{t}$, implying that the part $\zeta\left[x_{t}-\bar{x}\right]$ of the emission flow has to be sequestered. At $t^{\prime}$, the inverse demand function is going between the two steps of the marginal cost curve at $\bar{x}$, implying that the optimal consumption must amount to $\bar{x}$ and that abating is too costly. At time $t^{\prime \prime}$, the inverse demand curve is crossing the lowest part of the marginal cost curve, so that the optimal consumption is lower than $\bar{x}$. Then, $Z_{t}$ is decreasing and the path is the Hotelling path forever.

To sum up, the optimal path consists in five phases as illustrated in Figure 3.

During the first phase $\left[0, t_{1}\right)$, the pollution stock is increasing, the resource price is equal to the full marginal cost, $p_{t}=c_{x}+\lambda_{0} e^{\rho t}-\mu_{0} e^{(\alpha+\rho) t} \zeta$, and only the fossil fuel is used: $q_{t}=x_{t}=d\left(p_{t}\right)$. At the end of the phase, $p_{t}=c_{x}+\lambda_{0} e^{\rho t}+c_{s} \zeta$, the marginal cost of a clean fuel consumption, and the ceiling is attained.

The second phase $\left[t_{1}, t_{2}\right)$ is a phase at the ceiling during which only the fossil resource is used and some part of the potential emission flow is sequestered: $p_{t}=c_{x}+\lambda_{0} e^{\rho t}+c_{s} \zeta$, $q_{t}=x_{t}=d\left(p_{t}\right)$ and $s_{t}=\zeta\left[d\left(p_{t}\right)-\bar{x}\right]$. At the end of the phase, $p_{t}=\bar{p}$. 
The third phase $\left[t_{2}, t_{3}\right)$ is still a phase at the ceiling during which $p_{t}=\bar{p}$, and only the fossil resource is consumed, $q_{t}=x_{t}=\bar{x}$, but the emission flow is no longer stockpiled, being just balanced by the natural regeneration. During this phase, $\left|\mu_{t}\right|$ is decreasing and becomes nil at the end of the phase.

The fourth phase $\left[t_{3}, t_{4}\right)$ is a pure Hotelling phase, $p_{t}=p_{t}^{H}\left(\lambda_{0}\right)$, only the fossil resource has to be used, $q_{t}=x_{t}=d\left(p_{t}\right)$ and since $x_{t}<\bar{x}$ the pollution stock starts to decrease. At the end of this phase, the energy price is just equal to the marginal cost of the renewable resource $c_{y}$ and the fossil resource is exhausted.

The last phase $\left[t_{4}, \infty\right)$ is a phase during which only the renewable resource is used: $q_{t}=y_{t}=\tilde{y}$ and $p_{t}=c_{y}$.

Figure 3 here

The values of the six variables $\lambda_{0}, \mu_{0}, t_{1}, t_{2}, t_{3}$ and $t_{4}$ characterizing such an optimal path are provided by solving the following six-equation system:

- The cumulative demand-supply balance equation for the fossil resource, which is now:

$$
\begin{aligned}
& \int_{0}^{t_{1}} d\left(c_{x}+\lambda_{0} e^{\rho t}-\mu_{0} e^{(\alpha+\rho) t} \zeta\right) d t+\int_{t_{1}}^{t_{2}} d\left(c_{x}+\lambda_{0} e^{\rho t}+c_{s} \zeta\right) d t \\
&+\left[t_{3}-t_{2}\right] \bar{x}+\int_{t_{3}}^{t_{4}} d\left(c_{x}+\lambda_{0} e^{\rho t}\right) d t=X^{0} .
\end{aligned}
$$

- The price continuity equation at $t_{1}$ :

$$
c_{x}+\lambda_{0} e^{\rho t_{1}}-\mu_{0} e^{(\alpha+\rho) t_{1}} \zeta=c_{x}+\lambda_{0} e^{\rho t_{1}}+c_{s} \zeta .
$$

- The pollution stock continuity equation at $t_{1}$ :

$$
Z^{0} e^{-\alpha t_{1}}+\zeta \int_{0}^{t_{1}} d\left(c_{x}+\lambda_{0} e^{\rho t}-\zeta \mu_{0} e^{(\alpha+\rho) t}\right) e^{-\alpha\left(t_{1}-t\right)} d t=\bar{Z} .
$$

- The price continuity equations at $t_{2}, t_{3}$ and $t_{4}$ :

$$
\begin{aligned}
& c_{x}+\lambda_{0} e^{\rho t_{2}}+c_{s} \zeta=\bar{p} \\
& c_{x}+\lambda_{0} e^{\rho t_{3}}=\bar{p} \\
& c_{x}+\lambda_{0} e^{\rho t_{4}}=c_{y} .
\end{aligned}
$$


We can now give a precise definition of a "large" reservoir. Consider a price and fossil resource exploitation paths that verify all the optimal conditions described above. Then, the stockpiled carbon mass, denoted by $C^{s}$, is:

$$
C^{s}=\zeta \int_{t_{1}}^{t_{2}}\left[d\left(c_{x}+\lambda_{0} e^{\rho t}+c_{s} \zeta\right)-\bar{x}\right] d t
$$

A reservoir is said to be large if $C^{s}<\bar{S}$. Then, society can effectively sequester as much as carbon as needed instead of releasing in the atmosphere, so that no rent has to be charged for the mere use of the reservoir.

In the following section, we examine the optimal paths in the case where such a carbon mass is larger than $\bar{S}$, the case of "small" sink capacity.

\section{The case of a single small reservoir}

If the sink capacity is limited, its use implies an opportunity cost in addition to the sequestration cost $c_{s}$. In other words, the "shadow cost" $\eta_{t}$ of $S_{t}$ cannot be nil. We know $(c f$. section 2) that, as long as the reservoir is not filled, the absolute value of $\eta_{t}$ is increasing at the social rate of discount: $S_{t}<\bar{S} \Rightarrow \eta_{t}=\eta_{0} e^{\rho t}$. Substituting for $\eta_{t}$ into condition (11) and given that $\gamma_{s t}=0$ provided $s_{t}>0$, we get:

$$
c_{s}=\eta_{0} e^{\rho t}-\mu_{t}-\bar{\gamma}_{s t} \Rightarrow-\mu_{t}=c s-\eta_{0} e^{\rho t}+\bar{\gamma}_{s t}
$$

Next, we can substitute for $-\mu_{t}$ into (12). Given that $\gamma_{x t}=0$ provided $x_{t}>0$, and after simplifications, we must have ${ }^{18}$ :

$$
u^{\prime}\left(x_{t}\right)=c_{x}+\lambda_{0} e^{\rho t}+\left(c_{s}-\eta_{0} e^{\rho t}\right) \zeta
$$

The full marginal cost of the fossil resource when at the ceiling, $Z_{t}=\bar{Z}$, and given that the sequestration capacity of the sink is not saturated, takes now the following expression:

$$
c_{m}= \begin{cases}c_{x}+\lambda_{0} e^{\rho t} & \text { if } x_{t}<\bar{x} \\ c_{x}+\lambda_{0} e^{\rho t}+\left(c_{s}-\eta_{0} e^{\rho t}\right) \zeta & \text { if } x_{t}>\bar{x}\end{cases}
$$

The optimal consumption path of the fossil resource for the active sequestration phase is similar to corresponding phase in Figure 3, excepted that $c_{x}+\lambda_{0} e^{\rho t}+c_{s} \zeta$ must be

\footnotetext{
${ }^{18}$ Remember that $\eta_{0}<0$ so that $c_{s}-\eta_{0} e^{\rho t}$, the full marginal cost of sequestration, is increasing over time.
} 
replaced by $c_{x}+\lambda_{0} e^{\rho t}+\left(c_{s}-\eta_{0} e^{\rho t}\right) \zeta$. As in the large reservoir case, only the part of the potential emission flow that exceeds the natural regeneration should be sequestered, that is:

$$
s_{t}=\zeta\left[d\left(c_{x}+\lambda_{0} e^{\rho t}+\left(c_{s}-\eta_{0} e^{\rho t}\right) \zeta\right)-\bar{x}\right]
$$

Since the full marginal cost, $c_{x}+\lambda_{0} e^{\rho t}+\left(c_{s}-\eta_{0} e^{\rho t}\right) \zeta$, is increasing over time, this sequestration phase should precede a phase during which the fossil resource exploitation is constrained by the natural regeneration capacity of the atmosphere at the ceiling, that is a phase during which $q_{t}=x_{t}=\bar{x}$ and $p_{t}=\bar{p}$ for $Z_{t}=\bar{Z}$.

As in the large reservoir case, the optimal path consists in five phases. However, the price path differs until the date at which sequestration is no longer optimal. The new optimal price path is illustrated in Figure 4.

Figure 4 here.

The five phases of the optimal trajectories are the following.

The first phase $\left[0, t_{1}\right)$ is a phase during which the ceiling is reached and only the fossil resource is used. The difference with the large reservoir cases is that, at the end of the phase, the energy price must be equal to $c_{x}+\lambda_{0} e^{\rho t}+\left(c_{s}-\eta_{0} e^{\rho t}\right) \zeta$ rather than to $c_{x}+\lambda_{0} e^{\rho t}+c_{s} \zeta$

The second phase $\left[t_{1}, t_{2}\right)$ is a phase at the ceiling with sequestration, similar to the second phase of the large reservoir case, except that the marginal additional cost of sequestration is now $c_{s}-\eta_{0} e^{\rho t}$ instead of $c_{s}$.

The other phases are strictly identical to the previous case since during these phases, sequestration is no longer active.

The values of the seven endogenous variables $\lambda_{0}, \mu_{0}, \eta_{0}, t_{1}, t_{2}, t_{3}$ and $t_{4}$ characterizing this type of optimal path are determined by solving the following seven-equation system:

- The cumulative fossil resource consumption-initial stock balance equation:

$$
\begin{array}{r}
\int_{0}^{t_{1}} d\left(c_{x}+\lambda_{0} e^{\rho t}-\mu_{0} e^{(\alpha+\rho) t} \zeta\right) d t+\int_{t_{1}}^{t_{2}} d\left(c_{x}+\lambda_{0} e^{\rho t}+\left(c_{s}-\eta_{0} e^{\rho t}\right) \zeta\right) d t \\
+\left[t_{3}-t_{2}\right] \bar{x}+\int_{t_{3}}^{t_{4}} d\left(c_{x}+\lambda_{0} e^{\rho t}\right) d t=X^{0}
\end{array}
$$


- The price continuity equation at $t_{1}$ :

$$
c_{x}+\lambda_{0} e^{\rho t_{1}}-\mu_{0} e^{(\alpha+\rho) t_{1}} \zeta=c_{x}+\lambda_{0} e^{\rho t_{1}}+\left(c_{s}-\eta_{0} e^{\rho t_{1}}\right) \zeta .
$$

- The pollution stock continuity equation at $t_{1}$ :

$$
Z^{0} e^{-\alpha t_{1}}+\zeta \int_{0}^{t_{1}} d\left(c_{x}+\lambda_{0} e^{\rho t}-\zeta \mu_{0} e^{(\alpha+\rho) t}\right) e^{\alpha\left(t-t_{1}\right)} d t=\bar{Z} .
$$

- The price continuity equation at $t_{2}$ :

$$
c_{x}+\lambda_{0} e^{\rho t_{2}}+\left(c_{s}-\eta_{0} e^{\rho t_{2}}\right) \zeta=\bar{p} .
$$

- The saturation equation of the carbon sink capacity at $t_{2}$ :

$$
\zeta \int_{t_{1}}^{t_{2}}\left[d\left(c_{x}+\lambda_{0} e^{\rho t}+\left(c_{s}-\eta_{0} e^{\rho t}\right) \zeta\right)-\bar{x}\right] d t=\bar{S} .
$$

- The price continuity equations at $t_{3}$ and $t_{4}$, similar to the ones coming from the large reservoir case:

$$
c_{x}+\lambda_{0} e^{\rho t_{3}}=\bar{p} \quad \text { and } \quad c_{x}+\lambda_{0} e^{\rho t_{4}}=c_{y}
$$

For these values of $\lambda_{0}, \mu_{0}, \eta_{0}, t_{1}, t_{2}, t_{3}$ and $t_{4}$, we demonstrate in Appendix A.2 that the other multipliers take values satisfying all the optimality conditions.

\section{The multiple reservoirs case}

We first examine the case of the optimal use of two reservoirs and generalize the analysis to any number of reservoirs.

\subsection{The problem setup}

Consider the case where two different sequestration devices can be used. Let us assume first that the least costly reservoir, indexed by 1 , is large. Since reservoir 2 will never be used, the analysis is the same than in section 4, i.e. in the case of a single large reservoir, but with a sequestration $\operatorname{cost} c_{s}=c_{s 1}$.

Next, let us assume that reservoir 1 is small in the sense that a rent $-\eta_{1 t}=-\eta_{10} e^{\rho t}$ would have to be charged for its use if only this reservoir was available. As in the small reservoir case, the two first phases of the optimal price paths would be $p_{t}=c_{x}+\lambda_{0} e^{\rho t}-$ 
$\mu_{0} e^{(\alpha+\rho) t} \zeta$ over $\left[0, t_{1}\right)$ and $p_{t}=c_{x}+\lambda_{0} e^{\rho t}-\mu_{0} e^{(\alpha+\rho) t} \zeta+\left(c_{s 1}-\eta_{10} e^{\rho t}\right) \zeta$ over $\left[t_{1}, t_{2}\right)$, with the following associated continuity conditions:

$$
c_{x}+\lambda_{0} e^{\rho t_{1}}-\mu_{0} e^{(\alpha+\rho) t_{1}} \zeta=c_{x}+\lambda_{0} e^{\rho t_{1}}-\mu_{0} e^{(\alpha+\rho) t_{1}} \zeta+\left(c_{s 1}-\eta_{10} e^{\rho t_{1}}\right) \zeta
$$

and

$$
c_{x}+\lambda_{0} e^{\rho t_{2}}-\mu_{0} e^{(\alpha+\rho) t_{2}} \zeta+\left(c_{s 1}-\eta_{10} e^{\rho t_{2}}\right) \zeta=\bar{p} .
$$

Consequently, we would follow a continuous and increasing marginal cost path, from $c_{x}+\lambda_{0}$ at $t=0$ up to $\bar{p}$ at $t_{2}$, and then:

- either $c_{x}+\lambda_{0} e^{\rho t}+c_{s 2} \zeta>c_{x}+\lambda_{0} e^{\rho t}+\left(c_{s 1}-\eta_{10} e^{\rho t}\right) \zeta$ over the interval $\left[t_{1}, t_{2}\right)$ and in this case, reservoir 2 will never be used despite the limited capacity of reservoir 1. The full marginal cost of the now "clean" fossil resource, owing to sequestration, into reservoir 1 would always be smaller than the marginal cost when sequestering into reservoir 2, even if for this last reservoir, the rent $\left|\eta_{2 t}\right|$ was nil;

- or there exists a date $\bar{t}<t_{2}$, such that:

$$
c_{x}+\lambda_{0} e^{\rho t_{1}}+c_{s 2} \zeta<c_{x}+\lambda_{0} e^{\rho t}+\left(c_{s 1}-\eta_{10} e^{\rho t}\right) \zeta, t<\bar{t}
$$

and in this case, reservoir 2 must also be used though the sequestration cost in this $\operatorname{sink}, c_{s 2}$, is larger than the sequestration cost in reservoir $1, c_{s 1}$.

Given this last possibility, we have to consider two alternatives: either reservoir 2 is "large", in a sense that will be defined later, or reservoir 2 is "small".

\subsection{The case of an additional large reservoir}

The second reservoir is said to be large if it allows for the carbon that is not already stockpiled in reservoir 1 to be effectively sequestered in reservoir 2, even if no rent is charged for the use of the capacity of this sink. Clearly, provided that the capacity of reservoir 1 is saturated, the time period during which sequestration in reservoirs 1 and 2 occurs, but also the carbon mass sequestered in reservoir 2, are endogenously determined.

The point to be noticed here is that, at the optimum, the two sinks should never be used simultaneously. If it was not the case, i.e. if $s_{i t}>0, i=1,2$ over an interval $\left(t^{\prime}, t^{\prime \prime}\right)$, 
$t^{\prime}<t^{\prime \prime}$, we would have $\gamma_{i t}=0$, and $\nu_{i t}=0,{ }^{19} i=1,2$ over the same interval and then, from (11):

$$
-\mu_{t}=c_{s i}-\eta_{i 0} e^{\rho t}+\bar{\gamma}_{s t}, t \in\left(t^{\prime}, t^{\prime \prime}\right), \quad i=1,2 .
$$

Substituting for $\mu_{t}$ in (12), we obtain:

$$
u^{\prime}\left(x_{t}+y_{t}\right)=c_{x}+\lambda_{0} e^{\rho t}+\left(c_{s i}-\eta_{i 0} e^{\rho t}\right) \zeta, t \in\left(t^{\prime}, t^{\prime \prime}\right), \quad i=1,2
$$

so that:

$$
c_{s 1}-\eta_{10} e^{\rho t}=c_{s 2}-\eta_{20} e^{\rho t}, t \in\left(t^{\prime}, t^{\prime \prime}\right)
$$

which is clearly impossible if $c_{s 1}<c_{s 2}$ for any $-\eta_{i 0} \geq 0, i=1,2$.

The only case in which we can get a phase during which the full marginal cost of the clean fossil resource by sequestering in reservoir 1 is smaller than the cost involved by sequestering in reservoir 2 and another phase during which the inverted inequality holds, is the case where $-\eta_{20}<-\eta_{10}$. Thus the stockpiling phase into reservoir 1 must always precede the stockpiling phase into reservoir 2, whatever the reservoir 2 capacity.

Taking this remark into account, the optimal path consists now in six phases:

- Phase $1,\left[0, t_{1}\right)$, rise of the pollution stock to the ceiling without any sequestration;

- Phase $2,\left[t_{1}, t_{2}\right)$, at the ceiling with active sequestration in reservoir 1 ;

- Phase $3,\left[t_{2}, t_{3}\right)$, at the ceiling with active sequestration in reservoir 2 ;

- Phase $4,\left[t_{3}, t_{4}\right)$, at the ceiling without any sequestration;

- Phase $5,\left[t_{4}, t_{5}\right)$, pure Hotelling path, with a pollution stock under the ceiling and forever;

- Phase $6,\left[t_{5}, \infty\right)$, renewable resource exploitation.

The values of the eight variables $\lambda_{0}, \mu_{0}, \eta_{10}, t_{1}, t_{2}, t_{3}, t_{4}$ and $t_{5}$ characterizing this type of path are determined by solving the following eight-equation system:

\footnotetext{
${ }^{19}$ If $s_{i t}>0$ over $\left(t^{\prime}, t^{\prime \prime}\right)$, then $S_{i t}<\bar{S}_{i}$ from which $\nu_{i t}=0$ and $\eta_{i t}=\eta_{i 0} e^{\rho t}$.
} 
- The cumulative fossil resource consumption-initial stock balance equation, here:

$$
\begin{gathered}
\int_{0}^{t_{1}} d\left(c_{x}+\lambda_{0} e^{\rho t}-\mu_{0} e^{(\alpha+\rho) t} \zeta\right) d t+\int_{t_{1}}^{t_{2}} d\left(c_{x}+\lambda_{0} e^{\rho t}+\left(c_{s 1}-\eta_{10} e^{\rho t}\right) \zeta\right) d t \\
+\int_{t_{2}}^{t_{3}} d\left(c_{x}+\lambda_{0} e^{\rho t}+c_{s 2} \zeta\right) d t+\left[t_{4}-t_{3}\right] \bar{x}+\int_{t_{4}}^{t_{5}} d\left(c_{x}+\lambda_{0} e^{\rho t}\right) d t=X^{0}
\end{gathered}
$$

- The price continuity equation at $t_{1}$ :

$$
c_{x}+\lambda_{0} e^{\rho t_{1}}-\mu_{0} e^{(\alpha+\rho) t_{1}} \zeta=c_{x}+\lambda_{0} e^{\rho t_{1}}+\left(c_{s 1}-\eta_{10} e^{\rho t_{1}}\right) \zeta .
$$

- The pollution stock continuity equation at $t_{1}$ :

$$
Z^{0} e^{-\alpha t_{1}}+\zeta \int_{0}^{t_{1}} d\left(c_{x}+\lambda_{0} e^{\rho t}-\zeta \mu_{0} e^{(\alpha+\rho) t}\right) e^{\alpha\left(t-t_{1}\right)} d t=\bar{Z} .
$$

- The price continuity equation at $t_{2}$ :

$$
c_{x}+\lambda_{0} e^{\rho t_{2}}+\left(c_{s 1}-\eta_{10} e^{\rho t_{2}}\right) \zeta=c_{x}+\lambda_{0} e^{\rho t_{2}}+c_{s 2} \zeta .
$$

- The saturation condition of reservoir 1 at $t_{2}$ :

$$
\zeta \int_{t_{1}}^{t_{2}}\left[d\left(c_{x}+\lambda_{0} e^{\rho t}\left(c_{s 1}-\eta_{1 t_{2}} e^{\rho t_{2}}\right) \zeta\right)-\bar{x}\right] d t=\bar{S}_{1}
$$

- The price continuity equations at $t_{3}, t_{4}$ and $t_{5}$ :

$$
\begin{aligned}
& c_{x}+\lambda_{0} e^{\rho t_{3}}+c_{s 2} \zeta=\bar{p}, \\
& c_{x}+\lambda_{0} e^{\rho t_{4}}=\bar{p}, \\
& c_{x}+\lambda_{0} e^{\rho t_{5}}=c_{y} .
\end{aligned}
$$

As mentioned above, "large" and "small" capacities are endogenous characteristics of the sinks. Reservoir 2 is said to be "large" if, given $\lambda_{0}, \mu_{0}, \eta_{10}, t_{1}, t_{2}, t_{3}, t_{4}$ and $t_{5}$ solutions of the equation system above, the carbon mass to be sequestered in this sink does not exceed its capacity:

$$
\zeta \int_{t_{1}}^{t_{2}}\left[d\left(c_{x}+\lambda_{0} e^{\rho t}+c_{s 2} \zeta\right)-\bar{x}\right] d t \leq \bar{S}_{2}
$$

Reservoir 2 will be said "small" if it does not allow for the sequestration of such a carbon mass. 


\subsection{The case of an additional small reservoir}

If the additional reservoir is small, a rent has to be charged for its use. The only difference with the previous case is that, during the phase of active sequestration into reservoir 2 , the full marginal cost of the clean fossil resource, i.e. its price, is $c_{x}+\lambda_{0} e^{\rho t}+\left(c_{s 2}-\eta_{20} e^{\rho t}\right) \zeta$.

The optimal path is characterized by determining the values of the nine variables $\lambda_{0}$, $\mu_{0}, \eta_{10}, \eta_{20}, t_{1}, t_{2}, t_{3}, t_{4}$ and $t_{5}$, solutions of a nine-equation system. Eight of those equations are the same as in the previous case, except that we must include a rent for the use of the reservoir 2 in the expression of the price path over $\left[t_{2}, t_{3}\right]$. In addition to these eight equations, we must add a saturation equation for reservoir 2 at $t_{3}$ :

$$
\zeta \int_{t_{2}}^{t_{3}}\left[d\left(c_{x}+\lambda_{0} e^{\rho t}+\left(c_{s 2}-\eta_{20} e^{\rho t}\right) \zeta\right)-\bar{x}\right] d t=\bar{S}_{2} .
$$

The corresponding optimal price path is illustrated in Figure 5.

Figure 5 here

\subsection{The case of $n$ reservoirs}

The previous analysis can be easily extended to the case of $n$ reservoirs. Let the sinks be indexed by strictly increasing order of sequestration costs: $c_{s 1}<\ldots<c_{s i}<\ldots<c_{s n}$. We assume that the $m$ first reservoirs, $m<n$, are together small in the sense that, if the sequestration opportunity can only be exercised by filling these $m$ reservoirs, the rent which is charged for the use of the $m^{\text {th }}$ reservoir, as well as the $m-1$ previous ones, is positive: $-\eta_{m 0}>0$. Reservoir $m$ should be used over $\left[t_{m}, t_{m+1}\right)$ and at the end of this sequestration phase, we must have $c_{x}+\lambda_{0} e^{\rho t_{m+1}}+\left(c_{s m}-\eta_{m 0} e^{\rho t_{m+1}}\right) \zeta=\bar{p}$. Hence, there are two alternatives:

- either $c_{x}+\lambda_{0} e^{\rho t}+c_{s m+1} \zeta \geq c_{x}+\lambda_{0} e^{\rho t}+\left(c_{s m}-\eta_{m 0} e^{\rho t}\right) \zeta$ over the interval $\left[t_{m}, t_{m+1}\right)$ and in this case, the $m+1^{\text {th }}$ reservoir should not be used;

- or there exists a date $\bar{t}<t_{m+1}$, such that:

$$
c_{x}+\lambda_{0} e^{\rho t}+c_{s m+1} \zeta<c_{x}+\lambda_{0} e^{\rho t}+\left(c_{s m}-\eta_{m 0} e^{\rho t}\right) \zeta, t>\bar{t}
$$

so that the $m+1^{\text {th }}$ sink should also be used. 
In the last case, either reservoir $m+1$ is "large", or it is "small", the definitions of a large and a small reservoir being the same than the ones established for reservoir 2 in section 6.2. If reservoir $m+1$ is large, the remaining sinks $m+2, \ldots, n$ should not be used and if not, we have to implement again the sequestration opportunity test as described above. For a finite number of reservoirs, we obtain an algorithm that converges to a finite step number.

\subsection{The model as a model of sequestration cost increasing with the cumulated sequestration}

Until now, we have assumed that the average cost of sequestration in each sink $i, c_{s i}$, is constant and thus equal to the marginal cost. Since the higher the amount of stored carbon the higher is the pressure in the reservoir, and since this high pressure makes the incremental carbon unit more costly to be stored, one has to assume that the sequestration cost is an increasing function of the carbon mass already injected. In other words, one may state that for each deposit $i, c_{s i}=c_{s i}\left(S_{i}\right)$ and $d c_{s i} / d S_{i}>0$, where $S_{i}$ is the carbon mass already stored into the sink $i$.

The model analyzed so far could be seen as a model where the functions $c_{s i}($.$) are$ approximated by step functions, the more numerous the steps, the more accurate the approximation. Let $m_{i}$ be the number of steps chosen to approximate the function $c_{s i}($. and let $1_{i}, \ldots, h_{i}, \ldots, m_{i}$ be the indexes of those various steps, by increasing order of average sequestration cost for each step, $c_{s i}^{1_{i}}<\ldots<c_{s i}^{h_{i}}<\ldots<c_{s i}^{m_{i}}, c_{s i}^{h_{i}}$ being the average (marginal) cost of sequestration at step $h_{i}$. Note that $\Delta \bar{S}_{i}^{h_{i}}$ is the absorption capacity for the step $h_{i}$ so that $\sum_{h_{i}=1}^{m_{i}} \Delta \bar{S}_{i}^{h_{i}}=\bar{S}_{i}$.

Would there be a single sequestration sink, the sink $i$, the identification to the preceding model is straightforward. The optimal exploitation rule for differentiated average sequestration costs prescribes that it is necessary to exploit them by increasing order of their costs. Let us apply this rule to the only $\operatorname{sink} i$, decomposed in $m_{i}$ differentiated sub-sinks. The rule in question leads to the first exploitation of the sub-sink whose cost is $c_{s i}^{1_{i}}$ and whose capacity is $\Delta S_{s i}^{1_{i}}$, then if necessary, the second sub-sink whose cost is $c_{s i}^{2_{i}}$ and whose capacity is $\Delta S_{s i}^{2_{i}}$, and so and so forth... In other words, it is necessary to exploit the sub-sinks in the natural order of filling the sink $i$. 
Let us consider now the case of $m$ different sinks, their respective cost functions being themselves approximated by a step function. Let us build the sequence of theoretical sinks $j=1, \ldots, m$ by sorting again the whole steps of the various sinks indexed by increasing order of their costs and by grouping the capacities of the steps whose costs are identical. We denote by $c_{s}^{j}$ the average cost of the theoretical $j$-ranked sink whose sequestration capacity is $\bar{S}^{j}: c_{s}^{1}<\ldots<c_{s}^{j}<\ldots<c_{s}^{m}$. Those theoretical sinks are built by recurrence as follows. For $j=1$ :

$$
c_{s}^{1}=\min \left\{c_{s}^{1 i}, i=1, \ldots, m\right\} .
$$

Let $I(1)$ be the set of indexes of sinks for which $c_{s}^{1 i}=c_{s}^{1}$. Then:

$$
\bar{S}^{1}=\sum_{i \in I(1)} \Delta \bar{S}_{i}^{1 i}
$$

Let us take out the steps whose $\operatorname{costs}$ are $c_{s}^{1}$ and start the procedure again. Since the cardinal of the set of steps is finite, the defined procedure includes a finite number of stages.

The number of theoretical sinks, $m$, is at most equal to $\sum_{i=1} m_{i}$. It would precisely be equal to this sum if the costs at the steps of the effective sinks were all differentiated.

The order by which one shall optimally use the theoretical sinks is the order of their costs. By proceeding in such a manner, the order of exploitation of the various steps of a single sink is the natural order of filling this reservoir. Moreover, all the effective steps that constitute a theoretical $j$-rank sink can be used simultaneously. At the optimum, the carbon shall thus be stored in several sinks at a time. Note that some part of a given reservoir may be used at some stage and next, other reservoirs used before going back the first one. Such "va et viens" between different deposits are neither generated by fixed costs as in Gaudet et al. (2001) or Hartwick et al. (1986), nor by different final uses of the fossil resource as in Chakravorty et al. (1994, 2005, 2006), nor by capacity restriction on the extraction rates of the non-renewable and of the renewable, as in Amigues et al. (1998), Favard (2002), Holland (2003), nor last for non-renewables having different pollution contents, as in Chakravorty et al. (2006-c) and Smulders et al. $(2005)^{20}$.

\footnotetext{
${ }^{20}$ On the order of extraction of non-renewable resources, see also Kemp and Long $(1980,1984)$ and for semi-renewable ones, see Gaudet et al. (2006).
} 


\section{Conclusion}

We have studied the economic rationale of capturing and sequestering the carbon, so as to maintain its atmospheric stock below some threshold level. Consuming a fossil resource, capturing and sequestering the pollution which is generated, is like consuming a resource coming simultaneously out of two mining sites: the proper underground site of extraction and the sequestering site, the rent to be charged for each one having to growth at the interest rate since both must satisfy the Hotelling arbitrage condition. In this paper, we insisted upon the multiplicity of sequestering sites. Clearly, a generalization along the Herfindahl (1967) line of analysis of the case of different mining sites is immediate. We have shown that such a policy ought to be implemented only once this critical level has been reached, whatever the cost of sequestration is, i.e. independently from the number of deposits, their access costs and their retention capacities.

Moreover, it stems from our analysis in section 6 that the optimal resources exploitation and the sequestration implementation, obtained with constant average costs, are robust to other costs functional specifications that would depend on the flow and/or the cumulative sequestered carbon and/or the resource extraction as far as the carbon deposit and the exhaustible resource are respectively concerned (see Heal, 1976).

The absence of sequestration in the short run does not mean the absence of environmental policy in the short run. On the contrary, even before its implementation, the sequestration option affects the optimal pace of the exhaustible resource exploitation that has to be reduced until the ceiling is reached. The consumption reduction is attributable both to the opportunity cost of emitted pollution before the ceiling and to the opportunity cost of pollution sequestration once the ceiling is reached, those costs adding up to the total delivery cost of the resource.

Finally, our definition of the storage process does not include the possibility of some leakage that would result in sending the carbon back to the atmosphere (see Herzog et al., 2003 and Paccala, 2003). This leakage phenomenon, would it be continuous over time, would not have any incidence on the optimal solution in the short run. In this case, only the length of the capture and storage phase would be extended to the entire phase at the ceiling, the sequestration activity exactly compensating the leakage at each date. 


\section{Appendix}

\section{A.1. Determining the solution of the constrained path: the case of no abatement opportunity}

The values of the five variables of the model, $\mu_{0}, \lambda_{0}, t_{1}, t_{2}$ and $t_{3}$ solve the following five-equation system:

- The cumulative demand of fossil fuel must balance the available stock:

$$
\int_{0}^{t_{1}} d\left(c_{x}+\lambda_{0} e^{\rho t}-\mu_{0} e^{(\alpha+\rho) t}\right) d t+\left[t_{2}-t_{1}\right] \bar{x}+\int_{t_{2}}^{t_{3}} d\left(c_{x}+\lambda_{0} e^{\rho t}\right) d t=X^{0}
$$

- The full marginal cost of the fossil fuel must be equal to the ceiling price $\bar{p}$ at $t_{1}$ :

$$
c_{x}+\lambda_{0} e^{\rho t_{1}}-\zeta \mu_{0} e^{(\alpha+\rho) t_{1}}=\bar{p} .
$$

- At $t_{1}$, the pollution stock is attaining the ceiling:

$$
Z^{0} e^{-\alpha t_{1}}+\zeta \int_{0}^{t_{1}} d\left(c_{x}+\lambda_{0} e^{\rho t}-\zeta \mu_{0} e^{(\alpha+\rho) t}\right) e^{-\alpha\left(t_{1}-t\right)} d t=\bar{Z}
$$

- At $t_{2}$, the shadow cost of the ceiling constraint must be nil, so that:

$$
c_{x}+\lambda_{0} e^{\rho t_{2}}=\bar{p}
$$

- At $t_{3}$, the end of the extraction period of the fossil resource, its price must choke the average cost of the clean renewable substitute:

$$
c_{x}+\lambda_{0} e^{\rho t_{3}}=c_{y}
$$

It can be easily checked that the values of $\mu_{0}, \lambda_{0}, t_{1}, t_{2}$ and $t_{3}$ solving the above system provide values of the other multipliers that satisfy all the optimality conditions (11)-(25) (see Chakravorty et al., 2006-a).

\section{A.2. Determining the solution of the constrained path: the case of a single small reservoir}

Let $p_{t}^{H}=c_{x}+\lambda_{0} e^{\rho t}$ be the Hotelling price path, $\hat{p}_{t}=p_{t}^{H}-\mu_{0} e^{(\alpha+\rho) t} \zeta$ the optimal price path preceding the ceiling phase and $\tilde{p}_{t}=p_{t}^{H}+\left(c_{s}-\eta_{0} e^{\rho t}\right) \zeta$, the optimal price path followed within the sequestration phase. In the single small reservoir case, the complete solution of program $(P)$ is: 
$x_{t}=\left\{\begin{array}{ll}d\left(\hat{p}_{t}\right) & , t \in\left[0, t_{1}\right) \\ d\left(\tilde{p}_{t}\right) & , t \in\left[t_{1}, t_{2}\right) \\ \bar{x} & , t \in\left[t_{2}, t_{3}\right) \\ d\left(p_{t}^{H}\right) & , t \in\left[t_{3}, t_{4}\right) \\ 0 & , t \in\left[t_{4}, \infty\right)\end{array}, y_{t}=\left\{\begin{array}{ll}0 & , t \in\left[0, t_{4}\right) \\ \tilde{y} & , t \in\left[t_{4}, \infty\right)\end{array}, s_{t}= \begin{cases}0 & , t \notin\left[t_{1}, t_{2}\right) \\ \zeta\left[d\left(\tilde{p}_{t}\right)-\bar{x}\right] & , t \in\left[t_{1}, t_{2}\right)\end{cases}\right.\right.$

Associated Lagrange multipliers are:

$$
\begin{aligned}
& \gamma_{s t}=\left\{\begin{array}{ll}
c_{s}+\mu_{0} e^{(\alpha+\rho) t}-\eta_{0} e^{\rho t} & , t \in\left[0, t_{1}\right) \\
0 & , t \in\left[t_{1}, t_{2}\right) \\
c_{s}+\left(p_{t}^{H}-\bar{p}\right) / \zeta & , t \in\left[t_{2}, t_{3}\right) \\
c_{s} & , t \in\left[t_{3}, \infty\right)
\end{array}, \bar{\gamma}_{s t}=0, t \geq 0\right. \\
& \gamma_{x t}= \begin{cases}0 & , t \in\left[0, t_{4}\right) \\
p_{t}^{H}-c_{y} & , t \in\left[t_{4}, \infty\right)\end{cases} \\
& \gamma_{y t}= \begin{cases}c_{y}-\hat{p}_{t} & , t \in\left[0, t_{1}\right) \\
c_{y}-\tilde{p}_{t} & , t \in\left[t_{1}, t_{2}\right) \\
c_{y}-\bar{p} & , t \in\left[t_{2}, t_{3}\right) \\
c_{y}-p_{t}^{H} & , t \in\left[t_{3}, t_{4}\right) \\
0 & , t \in\left[t_{4}, \infty\right)\end{cases} \\
& \eta_{t}=\left\{\begin{array}{ll}
\eta_{0} e^{\rho t} & , t \in\left[0, t_{2}\right) \\
0 & , t \in\left[t_{2}, \infty\right)
\end{array} \quad, \nu_{S t}=0, t \geq 0\right. \\
& \mu_{t}= \begin{cases}\mu_{0} e^{(\alpha+\rho) t} & , t \in\left[0, t_{1}\right) \\
\eta_{0} e^{\rho t}-c_{s} & , t \in\left[t_{1}, t_{2}\right) \\
\left(p_{t}^{H}-\bar{p}\right) / \zeta & , t \in\left[t_{2}, t_{3}\right) \\
0 & , t \in\left[t_{3}, \infty\right)\end{cases} \\
& \nu_{Z t}= \begin{cases}0 & , t \in\left[0, t_{1}\right) \\
(\alpha+\rho) c_{s}-\alpha \eta_{0} e^{\rho t} & , t \in\left[t_{1}, t_{2}\right) \\
{\left[\dot{p}_{t}^{H}-(\alpha+\rho)\left(p_{t}^{H}-\bar{p}\right)\right] / \zeta} & , t \in\left[t_{2}, t_{3}\right) \\
0 & , t \in\left[t_{3}, \infty\right)\end{cases}
\end{aligned}
$$

Given (30), it is easy to check that if $\lambda_{0}, \mu_{0}, \eta_{0}, t_{1}, t_{2}, t_{3}$ and $t_{4}$ satisfy the system of seven equations described in section 5 , then the Lagrange multipliers defined by (31)-(36) are such that conditions (11)-(22) hold. In other respects, since the fossil resource stock $X_{t}$ is exhausted at $t_{4}$, the transversality condition (23) is satisfied. In the same way, since $\eta_{t}=0$ and $S_{t}=\bar{S}$ for $t \geq t_{2}$ on the one hand, $\mu_{t}=0$ and $Z_{t}=\bar{Z} e^{-\alpha\left(t-t_{4}\right)}$ for $t \geq t_{4}$ on the other hand, then conditions (24) and (25) are also satisfied. 


\section{References}

[1] Amigues J.P., P. Favard, G. Gaudet and M. Moreaux (1998). The optimal order of natural resource use when the capacity of the inexhaustible resource is limited, Journal of Economic Theory, 80(1), 153-170.

[2] Chakravorty U., E. Im and J. Roumasset (2006). Discontinuous extraction of a nonrenewable resource, Economics Letters, 90(1), 6-11.

[3] Chakravorty U. and D.L. Krulce (1994). Heterogeneous demand and order of resource extraction, Econometrica, 62(6), 1445-52.

[4] Chakravorty U., D.L. Krulce and J. Roumasset (2005). Specialization and nonrenewable resources: Ricardo meets Ricardo, Journal of Economic Dynamics and Control, 29(9), 1517-45.

[5] Chakravorty U., B. Magné and M. Moreaux (2006-a). Plafond de concentration atmosphérique en carbone et substitutions entre ressources énergétiques, Annales d'Économie et de Statistique, 81, 141-168.

[6] Chakravorty U., B. Magné and M. Moreaux (2006-b). A Hotelling model with a ceiling on the stock of pollution, Journal of Economic Dynamics and Control, 30(12), 28752904 .

[7] Chakravorty U., M. Moreaux and M. Tidball (2006-c). Ordering the extraction of polluting non-renewable resources, mimeo LERNA, University of Toulouse 1.

[8] Edmonds J., J. Clarke, J. Dooley, S.H. Kim and S.J. Smith (2004). Stabilization of $\mathrm{CO} 2$ in a B2 world: insights on the roles of carbon capture and disposal, hydrogen, and transportation technologies, Energy Economics, 26(4), 517-537.

[9] Favard P. (2002). Does productive capital affect the order of resource exploitation, Journal of Economic Dynamics and Control, 26(6), 911-918.

[10] Gaudet G., M. Moreaux and S. Salant (2001). Intertemporal depletion of resource sites by spatially-distributed users, American Economic Review, 91(4), 1149-1159.

[11] Gaudet G., M. Moreaux and C. Withagen (2006). The Alberta Dilemma: Optimal sharing of a water resource by an agricultural and oil sector, Journal of Environmental Economics and Management, 52(2), 548-566. 
[12] Gerlagh R. (2006). ITC in a global growth-climate model with CCS. The value of induced technical change for climate stabilization, The Energy Journal, Special issue on Induced Technological Change and Climate Change, 55-72.

[13] Gerlagh R. and B.C.C. van der Zwaan (2006). Options and instruments for a deep Cut in CO2 emissions: Carbon capture or renewables, taxes or subsidies?, The Energy Journal, 27(3), 25-48.

[14] Gitz V., P. Ambrosi, B. Magné and P. Ciais (2005). Is there an optimal timing for sequestration to stabilize future climate? American Geophysical Union, Geophysical Monograph, The Science and Technology of Carbon Sequestration: Assessment and Verification of Natural and Deliberate Carbon Sinks, American Geophysical Union Ed.

[15] Hartwick J.M., M.C. Kemp and N.V. Long (1986). Set-up costs and the theory of exhaustible resources, Journal of Environmental Economics and Management, 13(3), 212-224.

[16] Heal G. (1976). The relationship between price and extraction cost for a resource with a backstop technology, Bell Journal of Economics, 7(2), 371-378.

[17] Herfindahl O.L. (1967). Depletion and economic theory, in M. Gaffney, ed., Extractive Resources and Taxation, Madison: University of Wisconsin Press, 63-90.

[18] Herzog H., K. Caldeira and J.M. Reilly (2003). An issue of permanence: Assessing the effectiveness of temporary carbon storage, Climatic Change, 59(3), 293-310.

[19] Holland S.P. (2003). Extraction capacity and the optimal order of extraction, Journal of Environmental Economics and Management, 45(3), 569-588.

[20] Hotelling H. (1931). The economics of exhaustible resources, Journal of Political Economy, 39(2), 137-175.

[21] IPCC (2001). Climate Change 2001: The scientific basis, contribution of Working Group I to the third assessment report of the Intergovernmental Panel on Climate Change. Cambridge Univ. Press, Cambridge, UK. 
[22] IPCC (2005). Special report on carbon dioxide capture and storage, contribution of Working Group III, report of the Intergovernmental Panel on Climate Change. Cambridge Univ. Press, Cambridge, UK.

[23] Kemp M.C. and N.V. Long (1980). On two folk theorems concerning the extraction of exhaustible resources, Econometrica, 48(3), 663-673.

[24] Kemp M.C. and N.V. Long (1984). Toward a more general theory of the order of exploitation of non-renewable resource deposits, in: M.C. Kemp and N.V. Long (eds.), Essays on the economics of exhaustible resources, Elsevier Science Publishers, Amsterdam, 39-74.

[25] Kolstad C.D. and J.A. Krautkraemer (1993). Natural resource use and the environment in Handbook of Natural Resource and Energy Economics, Vol. III, Chap. 26, edited by A.V. Kneese and J.L. Sweeney, Elsevier Science Publishers.

[26] Kurosawa A. (2004). Carbon concentration target and technological choice, Energy Economics, 26(4), 675-684.

[27] Lafforgue G., B. Magné and M. Moreaux (2005). The optimal sequestration policy with a ceiling on the stock of carbon in the atmosphere, forthcoming in: R. Guesnerie and H. Tulkens (eds.), Conference volume of 6th CESifo Venice Summer Institute, David Bradford memorial conference on the design of climate policy, MIT Press, Cambridge.

[28] Lewis T.R. (1982). Sufficient conditions for extracting least cost resource first, Econometrica, 50(4), 1081-1083.

[29] McFarland J.R., H.J. Herzog and J.M. Reilly (2003). Economic modeling of the global adoption of carbon capture and sequestration technologies, In: J. Gale and Y. Kaya (eds.), Greenhouse Gas Control Technologies: Proceedings of the Sixth International Conference on Greenhouse Gas Control Technologies, Kyoto, Japan, Elsevier Science, Oxford, UK.

[30] Pacala S.W. (2003). Global Constraints on Reservoir Leakage, in Sixth International Greenhouse Gas Control Technologies Conference, edited by J. Gale, and Y. Kaya, pp. 477-482, Elsevier Science Unlimited, Kyoto (Japan). 
[31] Riahi K., E.S. Rubin, M.R. Taylor, L. Schrattenholzer and D. Hounshell (2004). Technological learning for carbon capture and sequestration technologies, Energy Economics, 26(4), 539-564.

[32] Smulders S. and E. Van der Werf (2005). Climate policy and the optimal extraction of fossil fuels, mimeo, Tilburg University. 


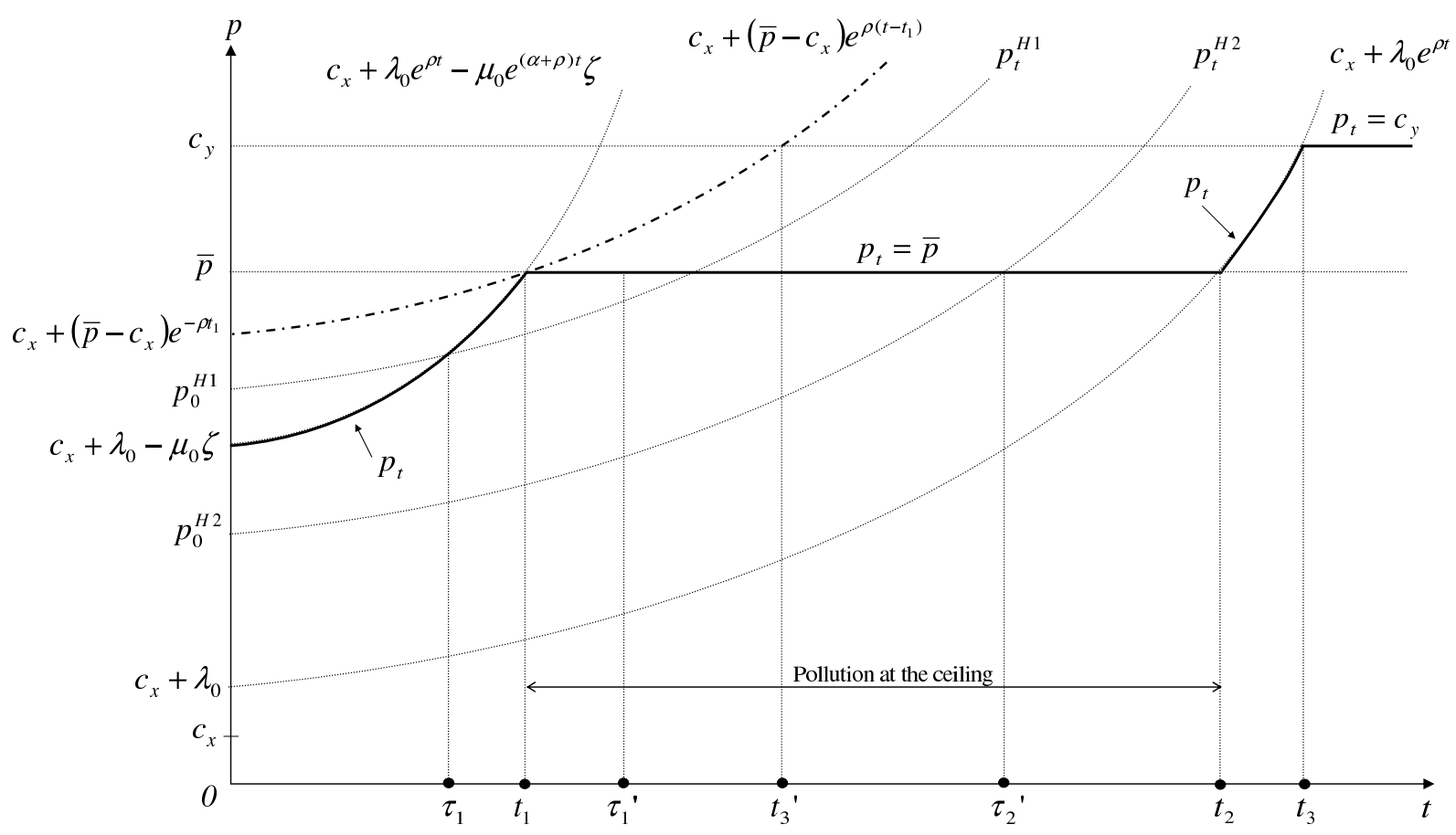

Figure 1: Optimal price path without any sequestration opportunity

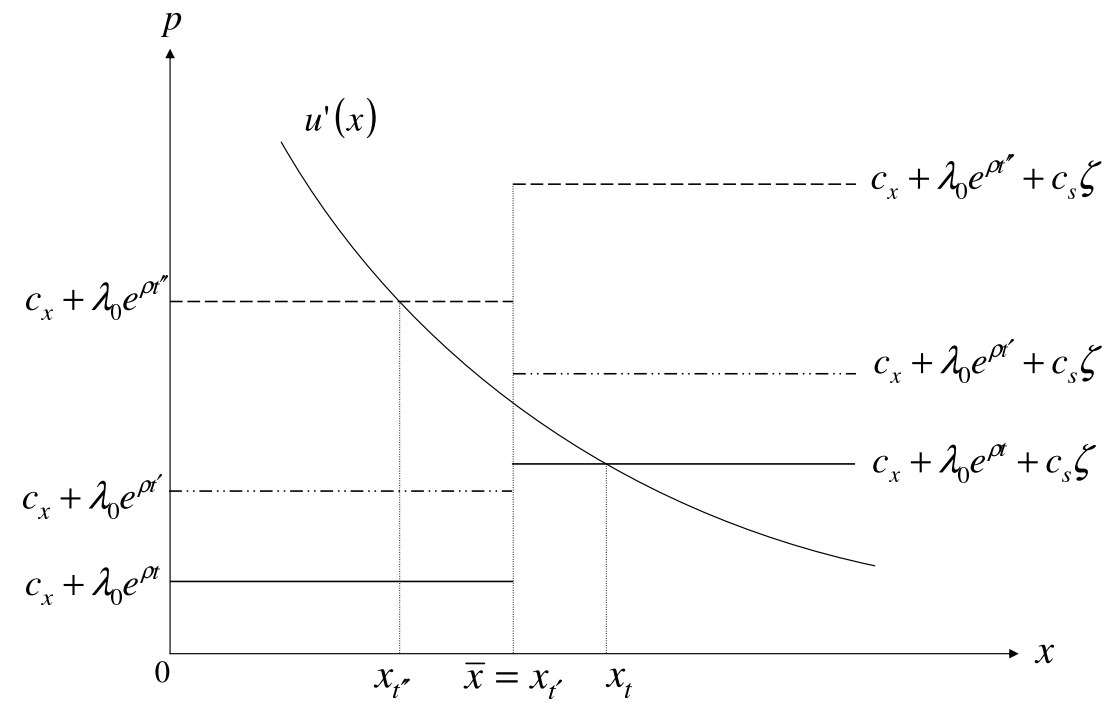

Figure 2: Full marginal cost of the fossil resource and optimal consumption when at the ceiling 


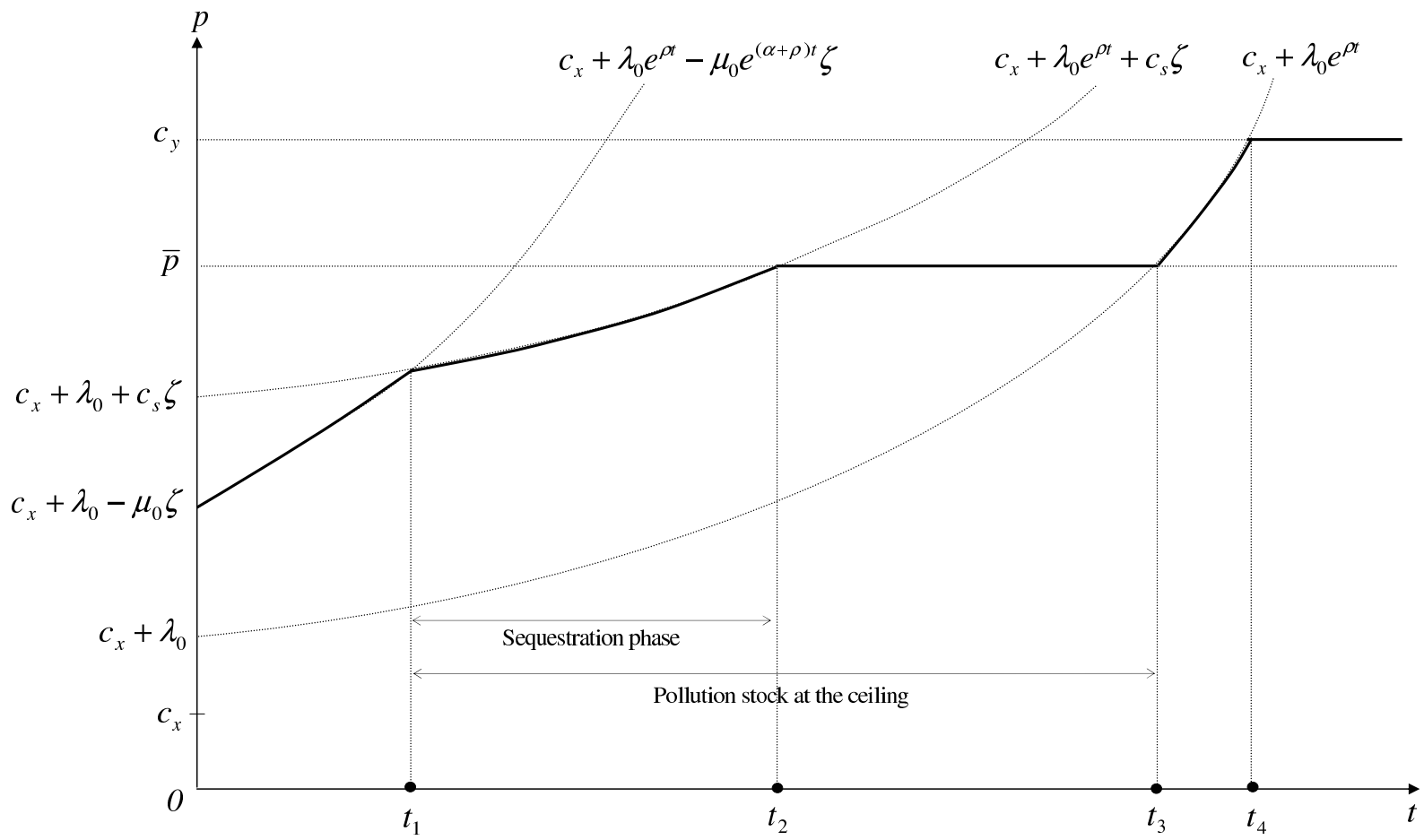

Figure 3: Optimal price path - The large reservoir case

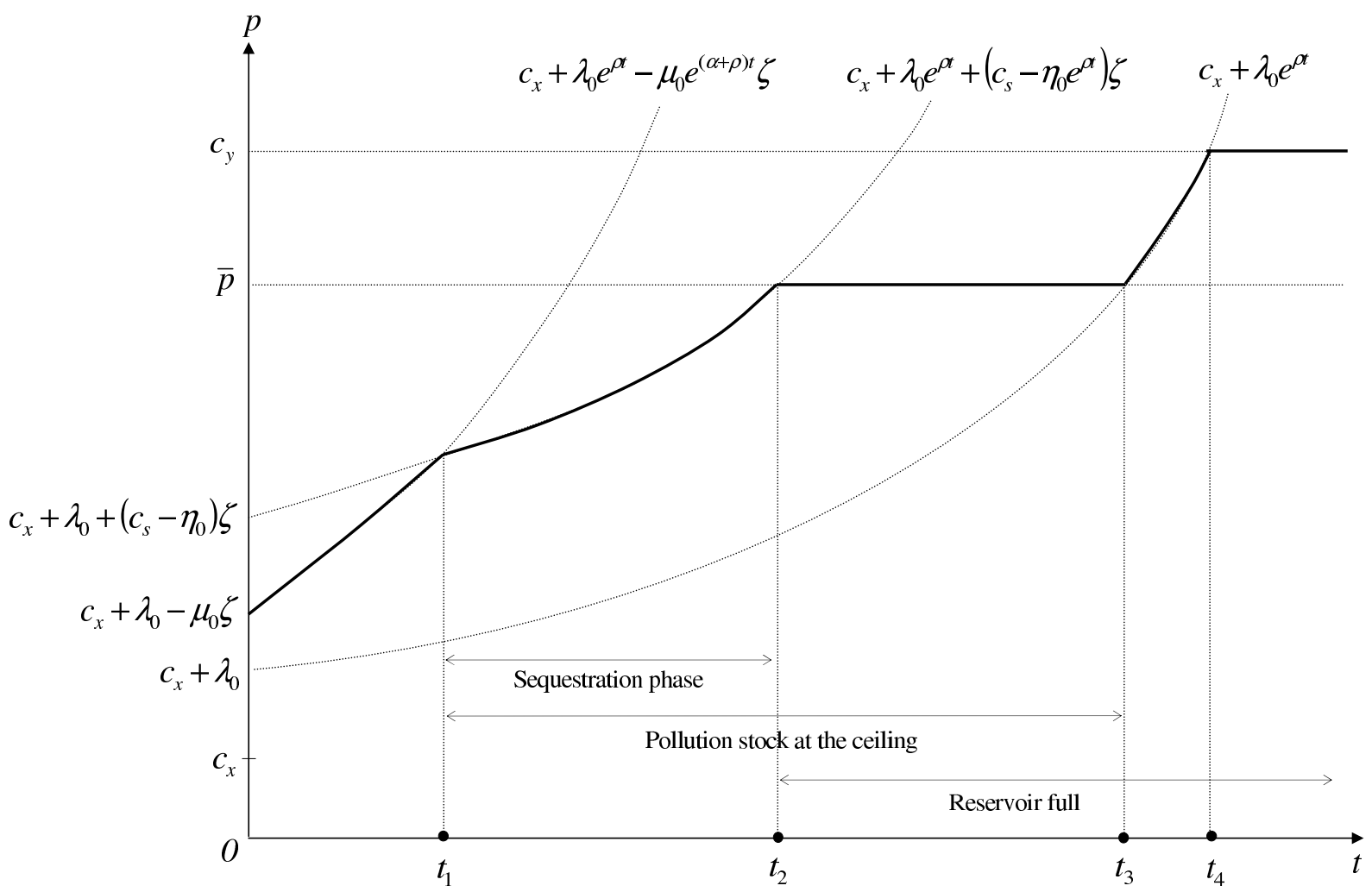

Figure 4: Optimal price path - The small reservoir case 


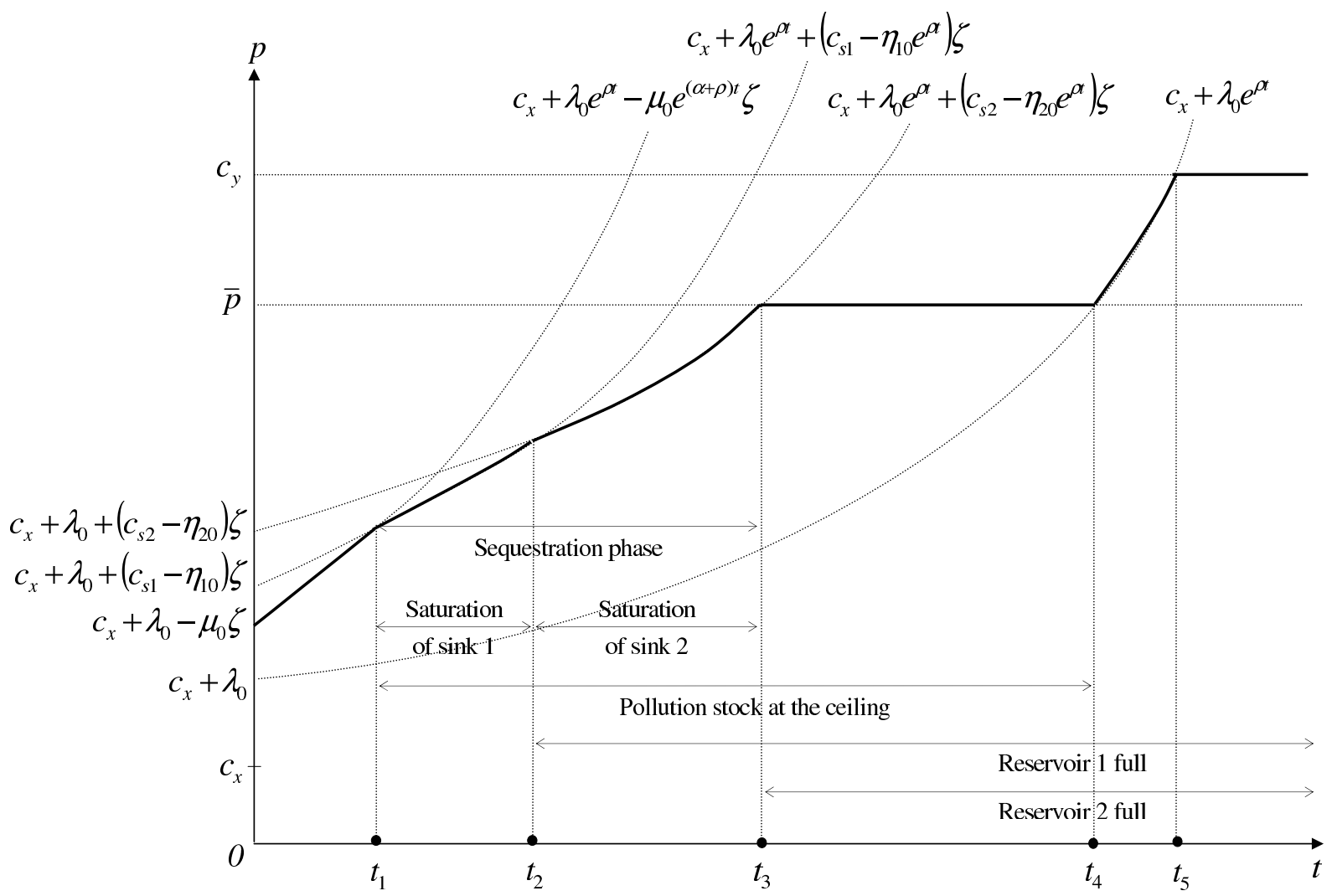

Figure 5: Optimal price path - The case of two small reservoirs 